\title{
Evaluación de las actitudes de estudiantes de maestros de educación infantil hacia la enseñanza y aprendizaje de la ciencia: validación de un cuestionario para el contexto español
}

\author{
Soraya Hamed ${ }^{*}$, Hortensia Morón-Monge y Ana Rivero \\ Facultad de Ciencias de la Educación, Dpto. de Didáctica de las Ciencias Experimentales y Sociales, Universidad de \\ Sevilla, Calle Pirotecnia s/n, 41013, Sevilla, España (correo-e: sha@us.es; hmoron@us.es; arivero@us.es).
}

${ }^{*}$ Autor a quien debe ser dirigida la correspondencia

Recibido Jul. 13, 2020; Aceptado Sep. 10, 2020; Versión final Nov. 10, 2020, Publicado Abr. 2021

\begin{abstract}
Resumen
El objetivo de este trabajo es validar un instrumento para analizar las actitudes de los futuros maestros de educación infantil sobre la enseñanza y aprendizaje de la ciencia de la Universidad de Sevilla, España. Para adaptar el instrumento al contexto español, se ha utilizado una metodología estandarizada de traducción. Además, se ha realizado una prueba piloto con 114 futuros maestros de educación infantil. Para la validez y confiabilidad del instrumento se realizaron los siguientes procedimientos: un análisis descriptivo, un análisis factorial (validez) y el coeficiente Alfa de Cronbach (fiabilidad) y de correlación intra-clase (estabilidad). Los resultados confirmaron las propiedades psicométricas para evaluar las actitudes de los futuros docentes en educación infantil sobre la enseñanza y aprendizaje de la ciencia en el contexto español. Se concluye que se dispone de un instrumento válido y confiable.
\end{abstract}

Palabras clave: maestros; validación; escala de actitudes; enseñanza; ciencias; educación infantil

\section{Assessment of kindergarten teachers' attitudes towards teaching and learning science: validation of a questionnaire for the Spanish context}

\begin{abstract}
The purpose of this research study is to validate an instrument to evaluate the attitudes towards science teaching and learning of kindergarten teachers in training at the University of Seville, Spain. To adapt the instrument to the Spanish context, a standardized translation methodology is used. In addition, a pilot study is performed with 114 prospective kindergarten teachers in training. For the validity and reliability of the instrument, the following procedures are performed: a descriptive analysis, a factor analysis (validity), and the Cronbach's alpha coefficient (reliability) and intra-class correlation (stability). The results confirm the instrument has the psychometric properties required to evaluate prospective kindergarten teacher' attitudes for science teaching and learning in the Spanish context. It is concluded that the instrument is valid and reliable.
\end{abstract}

Keywords: teacher training; validation; attitude scale; science teaching; early childhood education 


\section{INTRODUCCIÓN}

Vivimos en un mundo cada vez más complejo y cambiante en el que las personas deben formarse adecuadamente para enfrentarse a los retos socio-ambientales del siglo XXI. La actual crisis sanitaria por la COVID-19 y el momento de emergencia climática promulgada en el 2019, son solo dos ejemplos de las consecuencias del desequilibrio medioambiental y los problemas socio-ambientales del mundo de hoy. Por ello, se demanda un modelo educativo que permita dotar a las personas de herramientas para comprender su entorno e intervenir en él de manera fundamentada y productiva (Porlán, et al., 2020; Santos-González y Sarceda-Gorgoso, 2017).

Diferentes informes y evaluaciones nacionales e internacionales, tras analizar la situación actual de la educación científica (Confederación de Sociedades Científicas de España [COSCE], 2011; Rocard et al., 2007), abogan por la necesidad de un cambio curricular y formativo para que los escolares sepan actuar de manera informada y crítica ante los problemas de su vida cotidiana desde los primeros años de escolaridad. En este marco se encuentra el informe PISA (Informe del programa internacional para la Evaluación de Estudiantes (por sus siglas en inglés: Programme for International Student Assessment) y los proyectos SISCON (Science in a Social Context) y SATIS (Science and Technology in Society) de la ASE (Asociación para las ciencias de la educación) en los 80 que enfatizan la importancia de la enseñanza de las ciencias a partir de problemas o contextos reales (Donnelly y Ryder, 2011). Además, existen algunos estudios sobre la importancia de desarrollar un curriculum basado en competencias para la contextualización de los conocimientos desde la enseñanza de las ciencias (Pedrinacci, 2012; Contreras, 2016). Concretamente, nos referimos a una perspectiva de la enseñanza de las ciencias encaminada a la alfabetización ciudadana desde la acción para la resolución de problemas socio ambientales (Zeidler et al., 2005). Hacer frente a estos problemas requiere su comprensión, tomar decisiones y actuar, desde un planteamiento del curriculum orientado al activismo ciudadano (Hodson, 2014).

Teniendo presente esta perspectiva, los infantes son un potencial agente para el cambio que necesitamos. Sienten una gran curiosidad por su entorno más inmediato, al realizarse preguntas sobre el mundo, explorar y experimentar a partir de los sentidos (Eshach y Fried, 2005; Cantó et al., 2016; Mazas et al., 2018). Estas actitudes que poseen de forma natural hacia el aprendizaje de las ciencias, fomentan el desarrollo habilidades y competencias científicas como así recoge la legislación española (Gómez-Motilla y Ruíz-Gallardo, 2016). Por lo que es incuestionable la relación de las actitudes científicas sobre los procesos de enseñanzaaprendizaje (Osborne et al. 2003; Gómez-Motilla y Ruíz-Gallardo, 2016). Para ello, la enseñanza tiene que alinear con la forma en la cual el alumnado construye su conocimiento. En este sentido, la influencia de las actitudes hacia la ciencia y hacia el trabajo científico sobre su aprendizaje y, en el profesorado sobre su enseñanza, es de tal relevancia que deberían ser objetivos explícitos de la enseñanza. Sin embargo, para poder alcanzar esto, la enseñanza de las ciencias en la etapa de la educación infantil debe vencer hoy todavía muchos y diversos obstáculos (Lederman et al., 2019). El primero es la escasa valoración de las ciencias en educación infantil (Spector-Levy et al., 2013). Otros factores son la diversa interpretación que se hace del curriculum educativo en infantil, la falta de conocimiento por parte de los formadores de docentes en la enseñanza de las ciencias en esta etapa educativa (Lederman, et al., 2019); la escasez de investigaciones de didáctica de las ciencias sobre este grupo de estudio (Cantó et al., 2016). Estos son, entre otros, algunos de los principales retos a superar en la enseñanza e investigación de las ciencias en infantil.

Desde la formación del profesorado, también se detectan otros factores, particularmente las propias actitudes de los futuros maestros de educación infantil hacia las ciencias. Las creencias y actitudes de los docentes sobre su autoeficacia para la enseñanza, son algunos factores que tienen implicación en su praxis docente con los infantes (Gerde et al., 2018; Spector-Levy, et al., 2013). Para comprender las actitudes de los futuros maestros, es necesario conocer la actual situación de su formación inicial. En nuestro país, ésta ha ido mejorando en profesionalización con las diferentes reformas educativas, sin embargo, no alcanza los niveles de formación ni especialización que se exigen en otros países. El profesorado accede a la formación inicial con un amplio bagaje sobre lo que significa enseñar y aprender ciencias fruto de su experiencia como estudiantes, tratándose de un conocimiento alejado de lo que se propone desde la investigación educativa, lo cual repercute de forma relevante en su confianza y actitud hacia la enseñanza de la misma (Erden y Sönmez, 2011; McDonald et al., 2019; Oon et al., 2019). Por ello, no es de extrañar que existan actitudes tanto negativas como positivas hacia las ciencias en los maestros en formación (Mazas y Bravo, 2018; McDonald, Klieve y Kanasa, 2019). Particularmente, en la etapa de educación infantil se debe incidir en una formación docente que desarrolle competencias efectivas (González-Fernández et al., 2019; López et al., 2016; Rivero et al., 2020), pues estas implican una combinación de conocimientos, habilidades, valores, actitudes e intereses que conducen a una práctica profesional efectiva.

Además, se sabe poco sobre las actitudes y creencias de los maestros de educación infantil en formación inicial (Gómez-Motilla y Ruíz-Gallardo, 2016; Mazas y Bravo, 2018). En parte, porque se carece de 
instrumentos de medida válidos y fiables (Maier et al., 2013). Uno de los instrumentos más usados para valorar las actitudes de los futuros maestros de Educación Infantil hacia la ciencia son los cuestionarios con preguntas cerradas (de escala tipo Likert) y abiertas. Existen diferentes trabajos que lo evidencian (Spector-Levy et al., 2013; Sundberg y Ottander, 2013; Mazas y Bravo, 2018; Oon et al., 2019). La mayoría de ellos coinciden en que el profesorado se siente incómodo al planificar actividades científicas para involucrar al alumnado, pues tiene poca confianza en su capacidad como docente y se percibe a sí mismos con un conocimiento científico inadecuado (Oon et al., 2019; Spector-Levy et al., 2013), al tiempo de tener una percepción dogmática de la enseñanza de la ciencia (Sundberg y Ottander, 2013; Mazas y Bravo, 2018). Estos hallazgos tienen implicaciones significativas con respecto a cómo implementar actividades científicas en la educación infantil y de cómo alentar a los maestros a implicar a su alumnado en actividades científicas (Spector-Levy et al., 2013). Por ello, con este trabajo se pretende contribuir sobre esta temática a partir de la validación de un cuestionario para conocer las actitudes hacia las ciencias de los estudiantes de educación infantil en el contexto español.

\section{METODOLOGÍA}

El instrumento original utilizado en este trabajo se llama Teacher's Attitudes in Questionnaire (Spektor-Levy et al., (2013). Se compone de dos partes, una con preguntas abiertas y otra con preguntas cerradas tipo Likert de cuatro valores. Esta última se caracteriza por medir el grado de acuerdo de 14 declaraciones formuladas tanto en positivo como en negativo y agrupadas en las siguientes dimensiones: 1. Actitud hacia la enseñanza de la ciencia, 2. Actitud hacia el conocimiento científico personal y 3 . Métodos para realizar actividades. En este trabajo se utiliza el instrumento tipo Likert con la misma finalidad que el inglés pero con algunas modificaciones (Tabla 5): se usa una escala de cinco valores (en lugar de cuatro), ya que posibilita al alumnado una opción central de neutralidad o inseguridad (Blanco y Alvarado, 2005). Además, las autoras contaban inicialmente con cinco ítems que excluyeron de su instrumento inicial para incrementar su fiabilidad. En este trabajo, se incluyeron para conseguir el mismo propósito. En total, el instrumento tiene 19 declaraciones. Finalmente, se ha incluido un apartado para que alumnado argumente el por qué de su elección. Sería, pues, la parte abierta del cuestionario. Con relación al procedimiento de validación, se efectuó siguiendo una metodología estandarizada basada en: la traducción por más de un traductor, retro-traducción (back-translation), desarrollo de un grupo de discusión y de una prueba piloto para evaluar su potencialidad. A continuación, se exponen las fases que caracterizan el proceso:

El proceso de traducción inicial se realizó de forma independiente y semántica por dos investigadores españoles que tienen un buen dominio de la lengua inglesa. Dichos investigadores contaron con la presencia de un tercer investigador miembro del equipo de investigación para contrastar las dos traducciones y crear una versión consensuada en español. Posteriormente, esta versión (versión 1) fue sometida a un proceso de retro-traducción al inglés por parte de dos investigadores de habla inglesa que dominan también la lengua española. Se contó, además, con un tercer investigador para comprobar la concordancia entre las preguntas de ambos instrumentos. Así, se obtuvo la siguiente versión consensuada (Versión 2). El siguiente paso fue la evaluación realizada por ocho alumnos internos del Departamento de Didáctica de las Ciencias de la Universidad de Sevilla. Se incluyen cuatro mujeres y cuatro hombres con una media de edad de 21 años. Son futuros maestros matriculados en el $2^{\circ}$ curso del Grado de Educación Primaria ajenos al estudio. Participaron de forma voluntaria para cumplimentar el cuestionario y discutir acerca del diseño y contenido del mismo. Para ello, se solicitó que puntuasen del 1 a 5 la pertinencia y claridad de cada ítem y realizaran los comentarios y sugerencias que considerasen necesarios.

Con respecto a la revisión de la traducción inicial, los tres investigadores comprobaron un 97\% de concordancia entre las preguntas de ambos instrumentos. Del mismo modo ocurrió en la retro-traducción. La reformulación parcial de algunos ítems estaba relacionada con cuestiones gramaticales más que de contenido. Una vez valorado, se realizó una prueba piloto con una muestra de 114 alumnos de 3 o curso del Grado de Educación Infantil para estudiar la potencialidad del instrumento. Se caracteriza por ser no probabilística de conveniencia por razones de accesibilidad. Lo conformaron estudiantes, principalmente mujeres (108 mujeres -90\%-), con una media de edad de 21 años matriculadas en el $3 \%$ curso del Grado de Educación Infantil de la Universidad de Sevilla, España (2018-2019). Durante su formación inicial, han cursado varias asignaturas relacionadas con la ciencia, tecnología y las matemáticas (Enseñanza del Entorno Natural en la Etapa de 0 a 6 años, Desarrollo del Pensamiento Matemático en Infantil y Tecnología de la Información y de la Comunicación Aplicadas a la Educación Infantil). Ninguno de los estudiantes ha ejercido en algún momento de maestro o se encuentra en ejercicio. La administración del instrumento se efectuó mediante papel y lápiz. Tardaron 30 minutos en cumplimentarlo y no tuvieron dificultades para responderlo.

Además, se utilizó el Análisis Factorial por componentes principales y mediante el método de rotación normalización Varimax para determinar la coherencia de los ítems respecto a las dimensiones (validez de constructo). Se valoró la pertinencia de su realización mediante las pruebas de adecuación muestral de 
Káiser-Meyer-Olkin (KMO) y la prueba de esfericidad de Barlett. La KMO es un índice que oscila entre 0 y 1 , de manera que si $\mathrm{KMO} \geq 0,75$ la idea de realizar un análisis factorial es buena, si 0,75> KMO $\geq 0,5$ la idea es aceptable y si $\mathrm{KMO}<0,5$ es inaceptable. Por otro lado, se precisa del test de esfericidad de Bartlett, que permite comprobar si las correlaciones entre las variables son significativas según el modelo de probabilidad Chi-cuadrado. Si el nivel crítico (Sig.) es menor que 0,05 , se podrá rechazar la hipótesis nula de esfericidad y, por consiguiente, asegurar la pertinencia de este tipo de análisis para explicar los datos. Para estimar la consistencia interna y la estabilidad del instrumento, se determinó el coeficiente Alfa de Cronbach (Cronbach, 1984) de los ítems que lo componen y el coeficiente de correlación intraclase (CCl), respectivamente. Este último, hace referencia a la repetitividad del instrumento. Los coeficientes pueden tomar valores entre 0 y 1. Se consideró un valor satisfactorio de alfa y de $\mathrm{CCl}$ superior a 0,7 . Asimismo, para analizar las preguntas del cuestionario, se realizan estadísticos descriptivos (porcentajes, medias, desviaciones típicas) y de análisis del contenido para analizar las respuestas de las preguntas abiertas, ya que permite enriquecer y/o complementar las anteriores. Todos los tratamientos cuantitativos se realizaron con el programa estadístico SPSS v.26.0 y el cualitativo (análisis del contenido, Mayring, 2014) con el programa ATLAS.ti v.8.

\section{RESULTADOS}

A continuación, y para mayor claridad, se presentan y analizan los resultados relativos al grupo de discusión y de la prueba piloto.

\section{Resultados obtenidos del grupo de discusión}

Las valoraciones fueron positivas para todos los estudiantes, con puntuaciones de 5 sobre 5 tanto en pertinencia como en claridad para todos los ítems. Realizaron la lectura individual del instrumento y hubo acuerdo total del mismo. No manifestaron la necesidad de realizar cambios de redacción ni de contenido, ya que todos los ítems fueron considerados comprensibles y precisos.

\section{Resultados obtenidos de la prueba piloto}

En relación con el análisis factorial, se pudo evidenciar cuatro componentes o factores claros que explican el $60,30 \%$ de la varianza (ver tabla 1 y 2 ) en lugar de los tres como se propone en el cuestionario original. El test de adecuación muestral de Kaiser-Meyer-Olkin y la prueba de esfericidad de Barlett mostraron resultados satisfactorios ya que el grado de asociación entre las variables es buena $(\mathrm{KMO}=0,75)$ y existe una correlación significativa entre las mismas ( $p$-valor $<0.001$ ). Las cargas factoriales fueron superiores a 0,50.

El primer factor (factor 1), con una varianza explicada del 19,65\% consta de las declaraciones asociadas a la Actitud hacia la enseñanza y aprendizaje de la ciencia con puntuaciones por encima de 0,52. Con este factor, se constata la dimensión 1 del instrumento original en inglés, si bien se incluyen dos ítems nuevos que fueron excluidos en el citado instrumento (ítem 15 y 19). Es decir, se mantiene el ítem 1 y 2 relacionados con el compromiso de comenzar con temas científicos en Educación Infantil (ítem 1) y el reconocimiento de la capacidad de los niños para participar en actividades basadas en la investigación (ítem 2). También atiende a la participación e importancia de enseñar matemáticas, ciencias y tecnología en Educación infantil (ítem 15 y 19).

El segundo factor detectado (factor 2), con una varianza explicada del 15,33\%, incluye las 6 declaraciones relacionadas con la dimensión 3 referida a los Métodos para la realización de las actividades, con puntuaciones por encima de 0,56 . Es decir, se incluye la consideración de que la educación científica en Educación Infantil tiene un impacto a largo plazo en la actitud de los niños hacia las ciencias (ítem 10), la integración de multitud de actividades del dominio de las ciencias (ítem 3), matemáticas (ítem 7) y tecnología (ítem 12) y la satisfacción por el diseño y organización del aula y patio escolar (ítem 17 y 18, respectivamente). Si bien, se detecta una nueva declaración procedente del instrumento original (ítem 9) relacionada con la importancia de planificar actividades informáticas como parte del programa escolar. En este factor no se incluyen los ítem 5 y 14 relacionados con el compromiso con la educación ambiental y la búsqueda de actividades e información en internet.

El tercer factor (factor 3) es un factor nuevo, con una varianza explicada del $14,29 \%$, que lo denominamos Actitudes para mejorar el conocimiento y la enseñanza de la ciencia, conformado por 5 declaraciones saturadas por encima del 0,50 . Se incluye la necesidad de mejorar el conocimiento en el dominio de las ciencias (ítem 6), matemáticas (ítem 11) y tecnología (ítem 16) y el compromiso con la educación ambiental y la protección del medio ambiente (ítem 14) y la búsqueda de actividades e información en internet (ítem 5). El ítem 6 proviene de la dimensión 2 del instrumento original, el ítem 5 y 14 de la dimensión 3 y aparecen dos ítems nuevos relacionados con la necesidad de mejorar en dos dominios (en matemáticas y la tecnología) excluidos del instrumento original. 
El cuarto factor (factor 4), con una varianza explicada del 11,03\%, está conformado por declaraciones relacionadas con la dimensión 2 del cuestionario original: Actitud hacia el conocimiento científico personal con puntuaciones por encima del 0,58 . En este caso se incluye la confianza sobre el conocimiento científico y tecnológico propio (ítem 4 y 13, respectivamente) y la seguridad en la enseñanza de las matemáticas (ítem 8). En este factor, no se incluye el ítem 6.

Tabla 1. Varianza total explicada

\begin{tabular}{|l|l|l|l|}
\hline \multirow{2}{*}{ Componente } & \multicolumn{3}{|l|}{ Sumas de cargas al cuadrado de la rotación } \\
\cline { 2 - 4 } & Total & \% de varianza & $\%$ acumulado \\
\hline 1 & 3,734 & 19,653 & 19,653 \\
\hline 2 & 2,913 & 15,331 & 34,984 \\
\hline 3 & 2,715 & 14,289 & 49,273 \\
\hline 4 & 2,096 & 11,030 & 60,304 \\
\hline \multicolumn{2}{|l}{ Método de extracción: análisis de componentes principales } \\
\hline
\end{tabular}

Tabla 2. Matriz de componente rotado. Método de extracción: análisis de componentes principales. Método de rotación: Varimax con normalización Kaiser

\begin{tabular}{|c|c|c|c|c|}
\hline & \multicolumn{4}{|c|}{ Componente } \\
\hline & 1 & 2 & 3 & 4 \\
\hline $\begin{array}{l}\text { 19. Es importante enseñar matemáticas, ciencias y tecnología a niños en edad } \\
\text { preescolar }\end{array}$ & ,902 & - & - & - \\
\hline 1. El compromiso con los temas científicos debe comenzar en preescolar & ,863 & - & - & - \\
\hline $\begin{array}{l}\text { 2. Los niños en edad preescolar tienen la capacidad de participar en actividades basadas } \\
\text { en la investigación. }\end{array}$ & ,658 & - & - & - \\
\hline $\begin{array}{l}\text { 15. Me encantaría participar en asignaturas de matemáticas, ciencias y tecnología en } \\
\text { preescolar }\end{array}$ & ,525 & - & - & - \\
\hline 9. Es importante planificar actividades informáticas como parte del programa preescolar & - & 763 & - & - \\
\hline $\begin{array}{l}\text { 7.Integraría muchas actividades del dominio de las matemáticas en el programa } \\
\text { preescolar. }\end{array}$ & - & ,784 & - & - \\
\hline 17. Estoy satisfecho con la organización y el diseño del espacio de las aulas de infantil & - & ,713 & - & - \\
\hline 12. Integraría muchas actividades del campo de la tecnología en el programa preescolar & - & ,663 & - & - \\
\hline $\begin{array}{l}\text { 10. La educación científica en preescolar tiene un impacto a largo plazo en la actitud del } \\
\text { niño hacia las ciencias (unos pocos años) }\end{array}$ & - & ,663 & - & - \\
\hline 18. Estoy satisfecho con la organización del patio en la escuela & - & ,655 & - & - \\
\hline 3. Integraría muchas actividades del dominio de la ciencia en el programa preescolar & - & ,567 & - & - \\
\hline $\begin{array}{l}\text { 16. Necesito más conocimiento en el campo de la tecnología para poder participar en el } \\
\text { preescolar }\end{array}$ & - & - & ,709 & - \\
\hline $\begin{array}{l}\text { 14. Me comprometería con la educación ambiental y la protección del medio ambiente en } \\
\text { preescolar }\end{array}$ & - & - & ,787 & - \\
\hline $\begin{array}{l}\text { 11. Necesito más conocimiento en el dominio de las matemáticas para poder participar en } \\
\text { el preescolar }\end{array}$ & - & - & ,631 & - \\
\hline 6.Necesito más conocimiento en temas científicos. & & - & ,832 & - \\
\hline 5.Con frecuencia buscaría información y actividades para la escuela en Internet & - & - & ,504 & - \\
\hline 13. Me siento seguro con respecto a mi conocimiento en el campo de la tecnología & - & - & - & ,836 \\
\hline 4. Tengo confianza en mi conocimiento de las ciencias. & - & - & - & ,831 \\
\hline 8.Me siento seguro enseñando temas del dominio de las matemáticas. & - & - & - & ,681 \\
\hline
\end{tabular}

Los resultados obtenidos mediante el Alfa de Cronbach (ver tabla 3) indican una aceptable fiabilidad interna, ya que tanto las puntuaciones extraídas del instrumento en su conjunto $(\alpha=0,737$ y $\mathrm{CCl}=0,737, \mathrm{P}$ valor $=0,000<0,005$ ) como de cada ítem y dimensión (por encima de 0,63). Resultado similar al presentado en el instrumento original. Cabe señalar que los valores de alfa de Cronbach de las dimensiones 1 , 3 y 4 son por debajo de 0,7 debido al número reducido de ítems que incluye cada dimensión. 
Tabla 3. Estadísticos referidos a la adecuación de la muestra a las dimensiones y a la consistencia interna

\begin{tabular}{|l|l|c|c|}
\hline Factor & İtem & $\begin{array}{l}\text { Alfa de Cronbach } \\
\text { por ítem }\end{array}$ & $\begin{array}{l}\text { Alfa de Cronbach } \\
\text { por dimensión }\end{array}$ \\
\hline \multirow{2}{*}{$\begin{array}{l}\text { 1. Actitud hacia la } \\
\text { enseñanza y aprendizaje de }\end{array}$} & 1 & 0,734 &, 651 \\
\cline { 2 - 4 } & 2 & 0,725 & \\
\cline { 2 - 4 } & 15 & 0,702 & \\
\cline { 2 - 4 } & 19 & 0,722 &, 723 \\
\hline \multirow{2}{*}{$\begin{array}{l}\text { 2. Métodos para la } \\
\text { realización de las }\end{array}$ actividades } & 3 & 0,722 & \\
\cline { 2 - 4 } & 7 & 0,735 & \\
\cline { 2 - 4 } & 9 & 0,701 & \\
\cline { 2 - 4 } & 10 & 0,751 & \\
\cline { 2 - 4 } & 12 & 0,723 & \\
\cline { 2 - 4 } & 17 & 0,732 & \\
\hline \multirow{3}{*}{$\begin{array}{l}\text { 3. Actitudes para mejorar el } \\
\text { conocimiento y la }\end{array}$} & 18 & 0,712 & \\
enseñanza de la ciencia & 5 & 0,700 & \\
\cline { 2 - 4 } & 11 & 0,723 & \\
\cline { 2 - 4 } & 14 & 0,741 & \\
\cline { 2 - 4 } & 16 & 0,736 & \\
\hline \multirow{3}{*}{$\begin{array}{l}\text { 4. Actitud hacia el } \\
\text { conocimiento científico } \\
\text { personal }\end{array}$} & 4 & 0,726 & \\
\cline { 2 - 4 } & 8 & 0,745 & \\
\cline { 2 - 4 } & 13 & 0,737 & \\
\hline
\end{tabular}

Para facilitar el análisis, se agruparon los promedios y porcentajes de los valores extremos, de forma que: se considera que la muestra está de acuerdo con una declaración si los promedios son iguales o superiores al valor 4; está en desacuerdo si los promedios son iguales o inferiores al valor 3 y se manifiesta inseguridad si los promedios son mayores de 3 y menores de 4 .

Tabla 4. Criterio de agrupación de los valores medios obtenidos en cada declaración en función del grado de acuerdo (A) y desacuerdo (D)

\begin{tabular}{|l|l|l|}
\hline De acuerdo $(A)$ & En desacuerdo $(D)$ & Inseguridad $(I)$ \\
\hline Promedio $\geq 4$ & Promedio $\leq 3$ & $3>$ Promedio $<4$ \\
\hline
\end{tabular}

Si analizamos las respuestas de los estudiantes, se detectan, en general, percepciones favorables sobre la actitud hacia la enseñanza y aprendizaje de la ciencia, hacia el conocimiento científico personal y los métodos para realizar las actividades (ver tabla 5 y figura 1). Con respecto a la primera dimensión, la mayoría de los futuros maestros (por encima del $81 \%$ ) están de acuerdo con que se debe comenzar con temas científicos en Educación Infantil (ítem 1) y consideran que los niños tienen la capacidad de participar en actividades basadas en la investigación (ítem 2). La ciencia es considerada esencial para el desarrollo de los niños, ya que forma parte de su vida cotidiana, permite que puedan ser curiosos y comprender el mundo que les rodea y las actividades de investigación se consideran experiencias idóneas para lograrlo. Así lo declaran en la parte abierta: a) Los niños deben familiarizarse con temas científicos. El objetivo es darle sentido y comprender el mundo que les rodea y con ello ayudarlos a desarrollar de forma más efectiva y sistemática sus hallazgos, además ellos mismos suelen tener mucha curiosidad por los temas científicos y esto hace desarrollar sus potencialidades (Lorena); y b) Pienso que los niños/as de E.I aprenden y se desarrollan en interacción con el medio y de esta forma se produce el aprendizaje. Las actividades basadas en la investigación son una buena técnica, puesto que requieren de observación, manipulación, experimentación y reflexión. Los niños/as de E.I son investigadores o indagadores por naturaleza, tienen una curiosidad innata por experimentar y descubrir (Estrella R).

No obstante, un $14,1 \%$ duda de que los alumnos puedan investigar en el aula y el $19,2 \%$ de que se deba enseñar desde el inicio de dicha etapa (19,2\%). Las razones que lo explican son, fundamentalmente, la complejidad del área y la etapa educativa: i) No estoy segura si en Infantil se podría trabajar temas científicos, son difíciles (Gemma); ii) Pienso que son demasiado pequeños para aprender unos temas tan difíciles aun así algo si que se le puede introducir como algo de las plantas, el ciclo del agua (Lucía N); y iii) Si, son importantes, pero creo que no se les debe presionar ya que son asignaturas muy difíciles y en un futuro los niños pueden llegar a frustrarse (María). Con respecto a la Actitud hacia el conocimiento científico personal, percibimos que la mayoría (por encima del $80 \%$ ) tienen confianza en su conocimiento sobre la ciencia y tecnología (ítem 4 y 13, respectivamente) y en la enseñanza que realizarían de asignaturas como matemáticas (ítem 8). Sin embargo, el 95\% reconoce que necesitan más conocimiento en ambos temas para enseñarlos (ítem 6). Algunas de las razones destacadas se relacionan con la falta de formación, auto-confianza y experiencia para intervenir en el aula escolar. Veamos algunos ejemplos: a) El año pasado di la asignatura de Didáctica de las Matemáticas. Aprendí muchísimo, pero obviamente al no tener experiencia muchas cosas no las haría bien. Soy consciente de que me queda mucho por aprender todavía (Raquel); b) Este año he 
tenido Didáctica de la Tecnología de la Información. Pienso que se pueden hacer cosas muy divertidas, diferentes, originales y que a los niños les atraen mucho pero a día de hoy no podría ofrecer mucho a los niños sobre esto (Raquel); c) Sé de tecnología y de ciencias pero no como trabajarla con alumnos de preescolar (Gemma); y d) Ahora mismo no me sentiría preparada para tratar temas científicos en un aula de Educación Infantil por falta de experiencia y formación (Mirian).

Tabla 5. Estadísticos descriptivos por dimensión: porcentaje de desacuerdo (D), inseguridad (I) y acuerdo (A), media (M) y desviación típica (DS)

\begin{tabular}{|c|c|c|c|c|c|c|}
\hline Dimensión & Declaración & $D$ & 1 & $A$ & Media & DS \\
\hline \multirow{4}{*}{$\begin{array}{l}\text { 1: Actitud } \\
\text { hacia la } \\
\text { enseñanza de } \\
\text { la ciencia }\end{array}$} & $\begin{array}{l}\text { 1. El compromiso con los temas científicos debe comenzar en } \\
\text { preescolar }\end{array}$ & 4,5 & 14,7 & 81,7 & 4,02 & ,77 \\
\hline & $\begin{array}{l}\text { 2. Los niños en edad preescolar tienen la capacidad de } \\
\text { participar en actividades basadas en la investigación }\end{array}$ & 0 & 14,7 & 85,9 & 4,42 & ,73 \\
\hline & $\begin{array}{l}\text { 15. Me encantaría participar en asignaturas de matemáticas, } \\
\text { ciencias y tecnología en preescolar }\end{array}$ & 0 & 9,6 & 90,3 & 4,41 & ,66 \\
\hline & $\begin{array}{l}\text { 19. Es importante enseñar matemáticas, ciencias y tecnología } \\
\text { a niños en edad preescolar }\end{array}$ & 0 & 5,1 & 94,9 & 4,71 & ,56 \\
\hline \multirow{6}{*}{$\begin{array}{l}\text { 2: Métodos } \\
\text { para realizar } \\
\text { actividades }\end{array}$} & $\begin{array}{l}\text { 3. Integraría muchas actividades del dominio de la ciencia en } \\
\text { el programa preescolar }\end{array}$ & 0 & 24,4 & 75,7 & 3,86 & ,57 \\
\hline & $\begin{array}{l}\text { 9. Es importante planificar actividades informáticas como parte } \\
\text { del programa preescolar }\end{array}$ & 5,1 & 14,1 & 80,8 & 4,06 & ,81 \\
\hline & $\begin{array}{l}\text { 10. La educación científica en preescolar tiene un impacto a } \\
\text { largo plazo en la actitud del niño hacia las ciencias (unos } \\
\text { pocos años) }\end{array}$ & 0 & 9,6 & 90,3 & 4,41 & ,66 \\
\hline & $\begin{array}{l}\text { 12. Integraría muchas actividades del campo de la tecnología } \\
\text { en el programa preescolar }\end{array}$ & 5,1 & 14,7 & 80,1 & 3,90 & ,71 \\
\hline & $\begin{array}{l}\text { 17. Estoy satisfecho con la organización y el diseño del } \\
\text { espacio de las aulas de infantil }\end{array}$ & 5,1 & 14,7 & 80,1 & 3,90 & ,71 \\
\hline & $\begin{array}{l}\text { 18. Estoy satisfecho con la organización del patio en la } \\
\text { escuela }\end{array}$ & 0 & 5,1 & 94,8 & 4,71 &, 56 \\
\hline \multirow{5}{*}{$\begin{array}{l}\text { 3: Actitud para } \\
\text { mejorar el } \\
\text { conocimiento } \\
\text { y enseñanza } \\
\text { de la ciencia }\end{array}$} & $\begin{array}{l}\text { 5. Con frecuencia buscaría información y actividades para la } \\
\text { escuela en Internet }\end{array}$ & 5,1 & 34 & 60,9 & 3,71 & ,79 \\
\hline & 6. Necesito más conocimiento en temas científicos & 0 & 5,1 & 94,9 & 4,51 & 60 \\
\hline & $\begin{array}{l}\text { 7. Integraría muchas actividades del dominio de las } \\
\text { matemáticas en el programa preescolar }\end{array}$ & 24,4 & 25,6 & 50 & 3,36 & ,96 \\
\hline & $\begin{array}{l}\text { 11. Necesito más conocimiento en el dominio de las } \\
\text { matemáticas para poder participar en el preescolar }\end{array}$ & 0 & 25,6 & 74,4 & 3,94 & 67 \\
\hline & $\begin{array}{l}\text { 14. Me comprometería con la educación ambiental y la } \\
\text { protección del medio ambiente en preescolar }\end{array}$ & 19,8 & 29,5 & 50,7 & 3,41 & 1,08 \\
\hline \multirow{3}{*}{$\begin{array}{l}\text { 4. Actitud } \\
\text { hacia el } \\
\text { conocimiento } \\
\text { científico } \\
\text { personal }\end{array}$} & 4. Tengo confianza en mi conocimiento de las ciencias & 5,1 & 14,7 & 80,1 & 4,05 & ,81 \\
\hline & $\begin{array}{l}\text { 8. Me siento seguro enseñando temas del dominio de las } \\
\text { matemáticas }\end{array}$ & 0 & 9,6 & 90,4 & 4,21 & ,60 \\
\hline & $\begin{array}{l}\text { 13. Me siento seguro con respecto a mi conocimiento en el } \\
\text { campo de la tecnología }\end{array}$ & 5,1 & 14,1 & 80,8 & 4,06 & ,81 \\
\hline
\end{tabular}

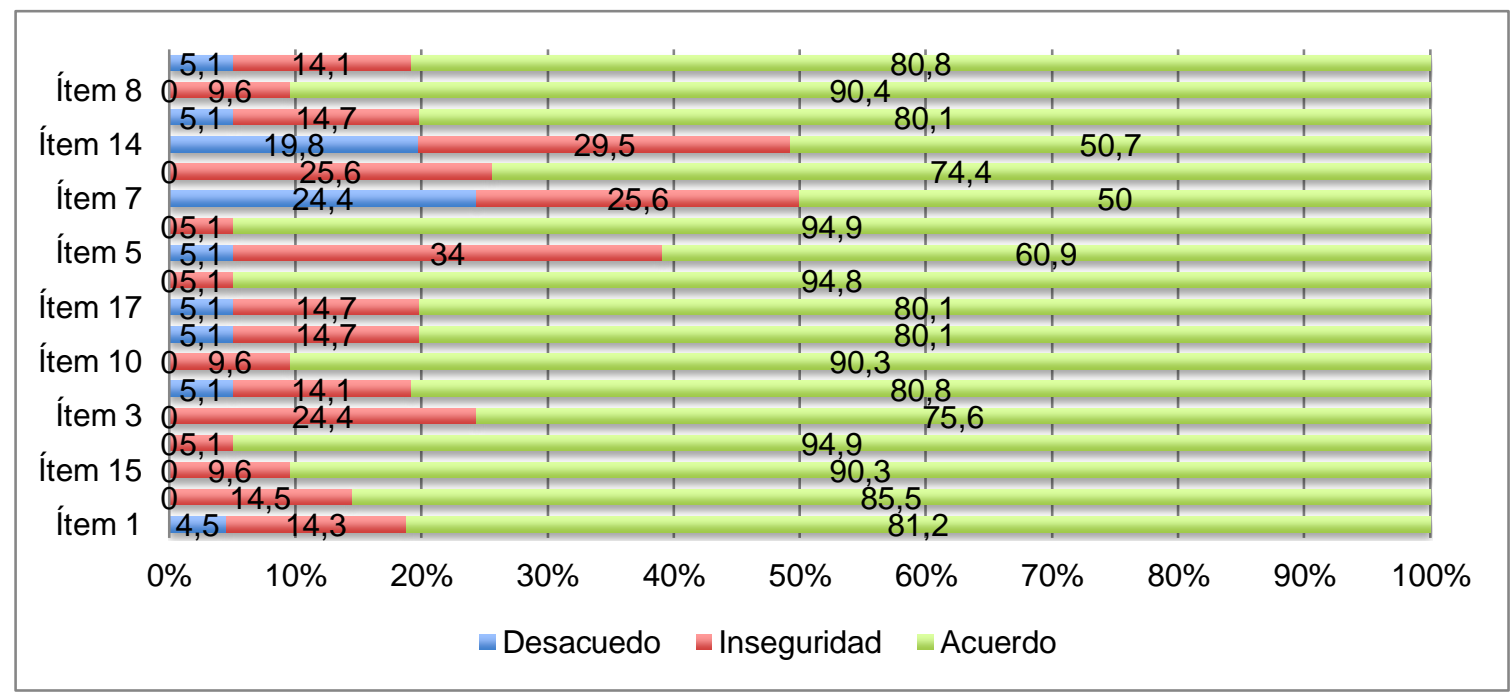

Fig. 1. Estadísticos descriptivos (porcentaje de desacuerdo, inseguridad y acuerdo) por ítem y dimensión 
Y, finalmente, con respecto a los Métodos para realizar las actividades, la mayoría de los participantes (por encima del 74\%) consideran que la educación científica tiene un impacto a largo plazo en la actitud de los niños (ítem 10), integrarían actividades relacionadas con las ciencias y la tecnología (ítem 3 y 12) y manifiestan su conformidad con el diseño y organización de las aulas en Infantil (ítem 17 y 18) salvo excepciones. Algunos señalan que las aulas están mal organizadas o siguen siendo "tradicionales", lo que dificulta la realización de diversas actividades. Por ejemplo: a) Todo lo que se aprende a conciencia de pequeño tiene una repercusión en el futuro, obviamente si se trabaja desde pequeño hasta secundaria, por ejemplo, garantizamos que ese impacto realmente se va a producir (EstrellaG); b) Siempre es bueno que integrar actividades de todos los ámbitos en educación infantil, ya que los niños deben tener conocimiento de todo (Mónica); c) Hay veces que los patios de algunas escuelas no están bien organizados, ya que los niños están en constante exploración y hay patios que limitan esa exploración (Mireya); y d) Para nada. Creo que todavía es todo MUY tradicional. Se necesita ya un cambio. Más zonas habilitadas para los rincones para que los niños puedan elegir qué les gusta hacer, zonas de descanso, zona para la lectura y no tantas mesas y sillas ordenadas frente a la pizarra (Raquel).

Sin embargo, se detecta que la mitad de los participantes no opinan igual con las actividades de matemáticas, ya que se muestran divididos entre el desacuerdo y la inseguridad (D: 24,4\%; I: 25,6\%; media:3,36; ds:0,96; ítem:7). En el caso de las actividades de ciencias, se localiza un $25,6 \%$ que no las integrarían. Un $29,5 \%$ dudan o están en desacuerdo $(19,8 \%)$ en su compromiso con la educación ambiental y protección del medio ambiente en Infantil (media: 3,41; ds:1,08; ítem:14) y sobre la búsqueda frecuente de actividades en internet (D: 5,1\%; I: 34\%; media:3,71; ds:0,79; ítem:5). Se vuelve aludir a la falta formación y el hecho de mantener el equilibrio de las actividades con relación a la temática. Por ejemplo: a) Integraría bastantes actividades, pero sin excesos, sin restarle importancia a otras asignaturas y sin agobiar a los alumnos. (Raquel); b) Integraría diferentes actividades del dominio de la ciencia y tecnología y a todas las diferentes áreas educativas (Manuel); c) Al igual que con las ciencias, las matemáticas es un ámbito que no se ha tocado demasiado durante la carrera por lo que, aunque tengamos conocimientos de matemáticas sobre lo que hemos estudiado en otras etapas educativas (Julia); d) El medio ambiente y su protección es un tema que apenas he trabajado durante mi formación (Luis); e) Internet sería un apoyo más, no el único (Mirian); y f) Internet es una buena herramienta. Además de libros, enciclopedias (Ana). En definitiva, el instrumento partía de tres dimensiones provenientes de la versión original de instrumento (en inglés) y, de acuerdo a los resultados obtenidos, parece indicar que podríamos tener cuatro dimensiones en lugar de tres. En la Tabla 6 se presenta el instrumento definitivo como resultado de este trabajo.

Tabla 6. Instrumento definitivo con las cuatro dimensiones (versión adaptada de Spector-Levy, Kesner Baruch y Mevarech, 2013)

Por favor, a continuación indica el grado de acuerdo o desacuerdo con cada declaración mediante un círculo los valores siguientes: totalmente en desacuerdo (TD), Desacuerdo (D), Inseguridad (I), Acuerdo (A), Totalmente de acuerdo (TA)

\begin{tabular}{|c|c|c|c|c|c|c|c|}
\hline Dimensión & Declaración & $T D$ & $D$ & I & $A$ & $T A$ & $\begin{array}{l}\text { Argumenta } \\
\text { por qué }\end{array}$ \\
\hline \multirow{4}{*}{$\begin{array}{l}\text { 1: Actitud hacia } \\
\text { la enseñanza } \\
\text { de la ciencia }\end{array}$} & $\begin{array}{l}\text { 1. El compromiso con los temas científicos debe } \\
\text { comenzar en preescolar }\end{array}$ & 1 & 2 & 3 & 4 & 5 & \\
\hline & $\begin{array}{l}\text { 2. Los niños en edad preescolar tienen la capacidad de } \\
\text { participar en actividades basadas en la investigación }\end{array}$ & 1 & 2 & 3 & 4 & 5 & \\
\hline & $\begin{array}{l}\text { 15. Me encantaría participar en asignaturas de } \\
\text { matemáticas, ciencias y tecnología en preescolar }\end{array}$ & 1 & 2 & 3 & 4 & 5 & \\
\hline & $\begin{array}{l}\text { 19. Es importante enseñar matemáticas, ciencias y } \\
\text { tecnología a niños en edad preescolar }\end{array}$ & 1 & 2 & 3 & 4 & 5 & \\
\hline \multirow{6}{*}{$\begin{array}{l}\text { 2: Métodos } \\
\text { para realizar } \\
\text { actividades }\end{array}$} & $\begin{array}{l}\text { 3. Integraría muchas actividades del dominio de la } \\
\text { ciencia en el programa preescolar }\end{array}$ & 1 & 2 & 3 & 4 & 5 & \\
\hline & $\begin{array}{l}\text { 9. Es importante planificar actividades informáticas } \\
\text { como parte del programa preescolar }\end{array}$ & 1 & 2 & 3 & 4 & 5 & \\
\hline & $\begin{array}{l}\text { 10. La educación científica en preescolar tiene un } \\
\text { impacto a largo plazo en la actitud del niño hacia las } \\
\text { ciencias (unos pocos años) }\end{array}$ & 1 & 2 & 3 & 4 & 5 & \\
\hline & $\begin{array}{l}\text { 12. Integraría muchas actividades del campo de la } \\
\text { tecnología en el programa preescolar }\end{array}$ & 1 & 2 & 3 & 4 & 5 & \\
\hline & $\begin{array}{l}\text { 17. Estoy satisfecho con la organización y el diseño del } \\
\text { espacio de las aulas de infantil }\end{array}$ & 1 & 2 & 3 & 4 & 5 & \\
\hline & $\begin{array}{l}\text { 18. Estoy satisfecho con la organización del patio en la } \\
\text { escuela }\end{array}$ & 1 & 2 & 3 & 4 & 5 & \\
\hline
\end{tabular}


Tabla 6: continuación

\begin{tabular}{|c|c|c|c|c|c|c|c|}
\hline Dimensión & Declaración & $T D$ & $D$ & I & $A$ & $T A$ & $\begin{array}{l}\text { Argumenta } \\
\text { por qué }\end{array}$ \\
\hline \multirow{5}{*}{$\begin{array}{l}\text { 3: Actitud para } \\
\text { mejorar el } \\
\text { conocimiento y } \\
\text { enseñanza de } \\
\text { la ciencia }\end{array}$} & $\begin{array}{l}\text { 5. Con frecuencia buscaría información y actividades } \\
\text { para la escuela en Internet }\end{array}$ & 1 & 2 & 3 & 4 & 5 & \\
\hline & 6. Necesito más conocimiento en temas científicos & 1 & 2 & 3 & 4 & 5 & \\
\hline & $\begin{array}{l}\text { 7. Integraría muchas actividades del dominio de las } \\
\text { matemáticas en el programa preescolar }\end{array}$ & 1 & 2 & 3 & 4 & 5 & \\
\hline & $\begin{array}{l}\text { 11. Necesito más conocimiento en el dominio de las } \\
\text { matemáticas para poder participar en el preescolar }\end{array}$ & 1 & 2 & 3 & 4 & 5 & \\
\hline & $\begin{array}{l}\text { 14. Me comprometería con la educación ambiental y la } \\
\text { protección del medio ambiente en preescolar }\end{array}$ & 1 & 2 & 3 & 4 & 5 & \\
\hline \multirow{3}{*}{$\begin{array}{l}\text { 4. Actitud hacia } \\
\text { el conocimiento } \\
\text { científico } \\
\text { personal }\end{array}$} & 4. Tengo confianza en mi conocimiento de las ciencias & 1 & 2 & 3 & 4 & 5 & \\
\hline & $\begin{array}{l}\text { 8. Me siento seguro enseñando temas del dominio de } \\
\text { las matemáticas }\end{array}$ & 1 & 2 & 3 & 4 & 5 & \\
\hline & $\begin{array}{l}\text { 13. Me siento seguro con respecto a mi conocimiento } \\
\text { en el campo de la tecnología }\end{array}$ & 1 & 2 & 3 & 4 & 5 & \\
\hline
\end{tabular}

\section{CONCLUSIONES}

Para finalizar, los resultados permiten señalar que: 1) se dispone de un instrumento válido y confiable para aplicarse y analizar las actitudes de los futuros maestros de educación infantil sobre la enseñanza y aprendizaje de la ciencia en el contexto español; 2) aunque se cuente con un proceso pormenorizado de traducción, conviene ser prudente con su uso para diferentes poblaciones de hispanohablantes, pues los resultados se limitan a un contexto determinado; 3) el análisis factorial pudo determinar la coherencia de los ítems de partida con la obtención de cuatro dimensiones principales (en lugar de tres). No obstante, se trata de un estudio preliminar de validación de algunos aspectos de la escala. Para continuar con la validación de constructo del instrumento se precisa realizar un análisis factorial confirmatorio con otra muestra diferente a la de este trabajo y más amplia $(\mathrm{N}>200)$; 4) los valores del coeficiente Alfa de Cronbach y del coeficiente de correlación intra-clase indican una aceptable confiabilidad del instrumento, tanto en su consistencia interna como en su estabilidad; 5) las declaraciones de los estudiantes sugieren la necesidad de reflexionar sobre qué estrategias serían las adecuadas para promover eficazmente el aprendizaje docente, en lo que se refiere a la construcción de sus conocimientos y actitudes favorables sobre la ciencia y su enseñanza; 6 ) las instituciones de formación docente deben poner a prueba las actitudes del profesorado en formación hacia la enseñanza de la ciencia junto con la ayuda de sus formadores y centros escolares colaborativos y 7) se precisa de la introducción de cursos más prácticos en los programas de preparación docente para que el alumnado tenga la oportunidad de probar sus propuestas formativas.

\section{REFERENCIAS}

Blanco, N., y Alvarado, M. E., Escala de actitud hacia el proceso de investigación científico social, Revista de Ciencias Sociales, 11(3), 537-544 (2005).

Cantó, J., Pro Bueno, A. y Solbes J., ¿Qué ciencias se enseñan y cómo se hace en las aulas de educación infantil? La visión de los maestros en formación inicial. http://dx.doi.org/10.5565/rev/ensciencias.1870, Enseñanza de las Ciencias, 34(3), 25-50 (2016)

Contreras, S.A., Pensamiento Pedagógico en la Enseñanza de las Ciencias: Análisis de las Creencias Curriculares y sus Implicancias para la Formación de Profesores de Enseñanza Media. doi: https://dx.doi.org/10.4067/S071850062016000100003, Formación Universitaria, 9(1), 15-24 (2016)

COSCE, Informe ENCIENDE. Enseñanza de las Ciencias en la Didáctica Escolar para edades tempranas en España. Ministerio de Ciencias e Innovación (2011)

Donnelly, J. y Ryder, J., The Pursuit of Humanity: Curriculum Change in English School Science. History of Education, 40(3), 291-313 (2011).

Erden, F. T. y Sönmez, S., Study of Turkish Preschool Teachers' Attitudes Toward Science Teaching. https://doi.org/10.1080/09500693.2010.511295, International Journal of Science Education, 33(8), 1149-1168 (2011).

Eshach, H. y Fried, M.N., Should Science be Taught in Early Childhood?. https://doi.org/10.1007/s10956-005-7198-9, Journal of Science Education and Technology, 14(3), 315-336 (2005).

Gerde, H. K., Pierce, S. J., Lee, K. y Van Egeren, L. A., Early childhood educators' self-efficacy in science, math, and literacy instruction and science practice in the classroom, doi: https://doi.org/10.1080/10409289.2017.1360127, Early Education and Development, 29(1), 70-90. (2018) 
Gómez-Motilla, C. y Ruiz-Gallardo, J. R., El rincón de la ciencia y la actitud hacia las ciencias en educación infantil http://doi.org/10.25267/Rev_Eureka_ensen_divulg_cienc.2016.v13.i3.10, Revista Eureka sobre enseñanza y divulgación de las ciencias, 13(3), 643-666 (2016).

González-Fernández, R., Zabalza-Cerdeirina, M.A., Medina-Domínguez, M. y Medina-Rivilla A., Modelo de Formación Inicial del Profesorado de Educación Infantil: Competencias y Creencias para su Capacitación, doi: http://dx.doi.org/10.4067/S0718-50062019000200083, Formación Universitaria, 12 (2), 83-96 (2019)

Hernández, V., Gómez, E., y otros siete autores, La actitud hacia la enseñanza y aprendizaje de la ciencia en alumnos de Enseñanza Básica y Media de la Provincia de Llanquihue,Región de Los Lagos-Chile. https://doi.org/10.4067/S071807052011000100004, Estudios Pedagógicos, 37 (1), 71-83 (2012)

Hodson, D. Becoming part of the solution: Learning about activism, learning through activism, learning from activism. In J. L. Bencze y S. Alsop (Eds.), Activist science and technology education (67-98). Dordrecht: Springer (2014)

Jiménez-Quintero, A.M., Sistematización de prácticas pedagógicas significativas en la carrera de licenciatura en educación infantil, http://doi.org/10.4067/S0718-50062020000400069, Formación Universitaria,13(4), 69-80 (2020)

Lederman, N.G., Abd-El-Khalick, F. y Smith, M.U. Teaching Nature of Scientific Knowledge to Kindergarten Through University Students, doi: https://doi.org/10.1007/s11191-019-00057-x, Science and Education, 28, 197-203 (2019)

López, C., Benedito, V. y León, M.J., El Enfoque de Competencias en la Formación Universitaria y su Impacto en la Evaluación: La Perspectiva de un Grupo de Profesionales Expertos en Pedagogía, doi: https://dx.doi.org/10.4067/S071850062016000400003, Formación Universitaria, 9(4), 11-22 (2016)

Maier, M. F., Greenfield, D. B. y Bulotsky-Shearer, R. J., Development and validation of a preschool teachers' attitudes and beliefs toward science teaching questionnaire, doi: https://doi.org/10.1016/j.ecresq.2012.09.003, Early Childhood Research Quarterly, 28(2), 366-378 (2013)

Mayring, P., Qualitative content analysis: theoretical foundation, basic procedures and software solution. Klagenfurt: Primary Publication (2014)

Mazas, B. y Bravo, B., Actitudes hacia la ciencia del profesorado en formación de educación infantil y educación primaria, Profesorado.https://doi.org/10.30827/profesorado.v22i2.7726, Revista de Currículum y Formación de Profesorado, 22 (2), 329 - 348 (2018)

Mazas, B., Gil-Quílez, M. J., Martínez-Peña, B., Hervás, A. y Muñoz, A., Los niños de infantil piensan, actúan y hablan sobre el comportamiento del aire y del agua, https://doi.org/10.5565/rev/ensciencias.2320, Enseñanza de las ciencias, 36 (1), 163-180 (2018)

McDonald, C. V., Klieve, H. y Kanasa, H., Exploring Australian Preservice Primary Teachers' Attitudes Toward Teaching Science Using the Dimensions of Attitude toward Science (DAS). https://doi.org/10.1007/s11165-019-09910-z, Research in Science Education (2019).

Oon, P.T., Hu, B. Y. y Wei, B., Early childhood educators' attitudes toward science teaching in Chinese schools. https://doi.org/10.1177/1836939119870890, Australasian Journal of Early Childhood, 44, (4), 423-435 (2019)

Osborne, J., Simon, S. y Collins, S., Attitudes towards science: A review of the literature and its implications, doi: https://doi.org/10.1080/0950069032000032199, International journal of science education 25(9), 1049-1079. (2003)

Pedrinacci, E., Caamaño, A., Cañal, P. y de Pro, A., 11 ideas clave: el desarrollo de la competencia científica. Barcelona: Grao (2012)

Porlán, P., Delord, G. Hamed, S. y Rivero, A., El cambio de las concepciones y emociones sobre la enseñanza a través de ciclos de mejora en el aula: un estudio con profesores universitarios de ciencias, doi: dx.doi.org/10.4067/S071850062020000400183, Formación Universitaria, 13(4), 183-200 (2020)

Rivero, S.E., Schmal, R.F. y Vidal-Silva, C.L., Fortaleza y debilidades de un programa para el desarrollo de competencias genéricas, doi: http://dx.doi.org/10.4067/S0718-50062020000400003, Formación Universitaria,13(4), 3-10 (2020)

Rocard, M., Science Education NOW: A renewed Pedagogy for the Future of Europe, Brussels: European Commission (2007)

Santos-González, C. y Sarceda-Gorgoso, C., Desarrollo de Competencias Docentes en Educacion Infantil. Una Experiencia Interdisciplinar en la Formacion Inicial de Profesores, doi: http://dx.doi.org/10.4067/S071850062017000600005, Formacion Universitaria, 10 (6), 2017.

Spektor-Levy, O., Kesner Baruch, Y. y Mevarech, Z., Science and scientific curiosity in pre-school-the teacher's point of view. https://doi.org/10.1080/09500693.2011.631608, International Journal of Science Education, 35, 2226-2253 (2013).

Sundberg, B., y Ottander, C., The Conflict Within the role: A longitudinal study of preschool student teachers' developing competence in and attitudes towards science teaching in relation to developing a professional role. Journal of Early Childhood Teacher Education, doi: https://doi.org/10.1080/10901027.2013.758540, 34(1), 80-94 (2013)

Zeidler, D. L., Sadler, T. D. y otros dos autores, Beyond STS: A research-based framework for socioscientific issues education. http://dx.doi.org/10.1002/sce.20048, Science Education, 89(3), 357-377 (2005) 